\title{
A Calculus for Stochastic Interventions: Causal Effect Identification and Surrogate Experiments
}

\author{
Juan D. Correa, Elias Bareinboim \\ Computer Science Department \\ Columbia University \\ $\{$ jdcorrea, eb $\} @$ cs.columbia.edu
}

\begin{abstract}
Some of the most prominent results in causal inference have been developed in the context of atomic interventions, following the semantics of the do-operator and the inferential power of the $d o$-calculus. In practice, many real-world settings require more complex types of interventions that cannot be represented by a simple atomic intervention. In this paper, we investigate a general class of interventions that covers some non-trivial types of policies (conditional and stochastic), which goes beyond the atomic class. Our goal is to develop general understanding and formal machinery to be able to reason about the effects of those policies, similar to the robust treatment developed to handle the atomic case. Specifically, in this paper, we introduce a new set of inference rules (akin to do-calculus) that can be used to derive claims about general interventions, which we call $\sigma$-calculus. We develop a systematic and efficient procedure for finding estimands of the effect of general policies as a function of the available observational and experimental distributions. We then prove that our algorithm and $\sigma$-calculus are both sound for the tasks of identification (Pearl, 1995) and z-identification (Bareinboim and Pearl, 2012) under this class of interventions.
\end{abstract}

\section{Introduction}

Causal relations are considered highly valuable and desirable throughout data-driven sciences due to their inherent interpretability and robustness to changing conditions. In machine learning, for example, they play a key role due to their amenability to extrapolation to new, unforeseen situations, and also their capability to support robust decision-making. Making sense of the world and constructing coherent and transparent explanations about it, almost invariably, hinge on our ability to learn and reason with cause and effect relationships (Pearl 2000; Spirtes, Glymour, and Scheines 2001; Bareinboim and Pearl 2016; Pearl and Mackenzie 2018).

One of the most common ways of learning about causal relations is through controlled experimentation. In practice, however, performing interventions is not always feasible due to its potentially harmful side effects, financial, and ethical considerations. This leads to one of the fundamental challenges in causal inference, namely, to determine whether the

Copyright (C) 2020, Association for the Advancement of Artificial Intelligence (www.aaai.org). All rights reserved. effect of an intervention can be computed without directly experimenting in the system, which is known as the problem of identification of causal effects (Pearl 2000, Def. 3.2.4). The most studied and well-understood type of intervention is called atomic, which is the most basic, simplest, and fundamental among many. In modern causal inference, atomic interventions are usually modeled through the $d o$-operator (Pearl 1995), which is denoted by $d o(X=x)$. Formally, $d o(X=x)$ represents the symbolic operation of replacing the underlying causal mechanism that naturally dictates the behavior of a variable $X$ with a constant value $x^{1}$.

The identification task relies on assumptions about the underlying causal system, which can be encoded in the form of a causal graphical model. For concreteness, consider the causal diagram in Fig. 1(a), where $X$ represents the choice to smoke, $W$ age, $Z$ a set of risk factors leading to tendency to smoke (e.g., peer pressure, education, SES, psychological age), and $Y$ the development or not of lung cancer. The task here is to compute the average effect of $X$ on $Y$ based on the observational (i.e., non-interventional) distribution $P(W, Z, X, Y)$. Using the $d o$-operator, this quantity can be formally written as $P(Y \mid d o(X=x))$, which describes the behavior of $Y$ when $X$ is fixed to $x$ (smoking or not) regardless of $Z$ or any other confounding factors.

Considering the example again, the difference between two do-distributions, $P(Y \mid d o(X=1))-P(Y \mid d o(X=0))$, amounts to the causal variability of $Y$ due to $X$, i.e., free of the influence of spurious factors. This difference can be thought of as a hypothetical regime corresponding to the new causal diagram as in Fig. 1(b), where the arrows incoming to $X$ are removed. Graphically, we will explicitly annotate the diagram with a regime node $\sigma_{X}$ to indicate that the

\footnotetext{
${ }^{1}$ This basic primitive has appeared in different contexts in the causal inference literature. It was introduced in econometrics by (Haavelmo 1943) and then (Strotz and Wold 1960). In the context of potential outcomes in statistics, it was formalized by (Neyman 1923) to describe randomized experiments, and then later on connected with observational studies by (Rubin 1974). In mathematical logic, it was discussed by (Lewis 1973) in the context counterfactuals in a possible-worlds semantics. It was given a more general and algorithmic treatment in artificial intelligence through the use of graphical models (Pearl 1993b; Spirtes, Glymour, and Scheines 1993).
} 
causal mechanism of $X$ has changed compared to the natural regime (this is a critical construct as discussed in Sec. 3).

There exists a body of literature concerned with identification of do-interventions from data collected under observational and experimental regimes, including celebrated results such as do-calculus (Pearl 1993a; 1995; 2000), and complete graphical and algorithmic conditions (Tian and Pearl 2002a; Tian 2004; Shpitser and Pearl 2006a; 2006b; Huang and Valtorta 2006; Bareinboim and Pearl 2012; Lee, Correa, and Bareinboim 2019).

While the intervention $d o(X=0)$ describes with mathematical precision a counterfactual world where smoking is banned from society, in practice, it is not likely that a policy could be implemented such that cigarettes would be completely wiped out from the streets. In words, we could eventually predict the effect of this new, idealized policy, however unlikely to be implemented in reality. This challenge has been a point subject to intense debate in causal inference circles (Woodward 2003; Heckman 2005; Cartwright 2007; Pearl 2010).

In this paper, we offer a mathematical solution to address this decade old debate. Going back to our example, for concreteness, policy-makers contemplate a more strict regulation on underage smoking and higher taxes on cigarettes sales that could be set in place. A sensible question in this context could be - what is the effect of a policy that inhibits smoking in people under 21 years of age, by $90 \%$ ? Such intervention is certainly non-atomic (which would entail that a $100 \%$ decrease in smoking should be enforced for this group), and in this case, the underlying mechanism for $X$ is replaced with a softer mechanism; these interventions are sometimes called soft or stochastic interventions.

Even though deciding the identifiability of complex interventions has been studied in the literature, there is still work to be done (Pearl 2000, Ch. 4). For instance, (Pearl and Robins 1995) studied the effect of interventions in longitudinal settings where the decision in each time step is dependant on the previous ones, which was called conditional plans. Further, other works investigated the effect of stochastic interventions, where the original causal mechanism of the treatment variable is replaced with a new known function (Dawid 2002; Didelez, Dawid, and Geneletti 2006; Tian 2008; Shpitser and Sherman 2018). For the case when the new function is unknown, the problem has been studied under the rubrics of transportability (Bareinboim and Pearl 2014; 2016; Correa and Bareinboim 2019).

Despite the high level of sophistication and generality achieved for reasoning with atomic interventions, we highlight the glaring difference with the non-atomic case. For instance, there exist no counterpart for $d o$-calculus in the non-atomic case nor general results on identifiability from experimental distributions produced by soft interventions. In this paper, we develop a general, symbolic, and algorithmic treatment for identifiability of arbitrary non-atomic interventions from both observational and experimental distributions. More specifically, our contributions are as follows:

1. Symbolic characterization. We introduce a set of inference rules, called $\sigma$-calculus, to reason about the effect of general types of intervention. Further, we provide a syn-
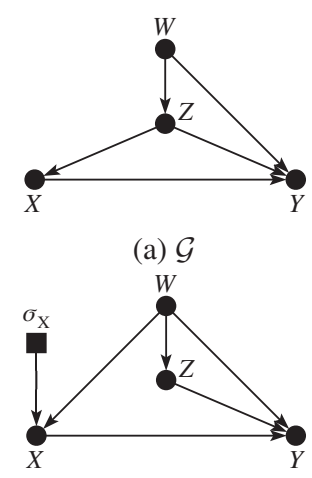

(c) $\mathcal{G}_{\sigma_{X}=g(w)}$

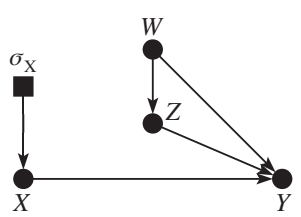

(b) $\mathcal{G}_{\sigma_{X}=d o(x)}$

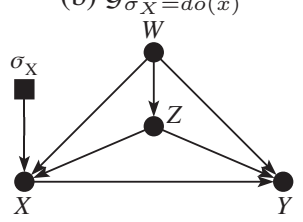

(d) $\mathcal{G}_{\sigma_{X}=P^{*}(x \mid z, w)}$
Figure 1: (a) original causal diagram $\mathcal{G}$. (b), (c), and (d) show the causal diagrams after an atomic, conditional, and stochastic intervention, respectively. See discussion in the introduction and examples 1 and 2 for details.

tactical method for deriving and verifying claims about such interventions given a causal graph.

2. Algorithmic solution. We develop an efficient procedure to determine the identifiability of the (conditional) effect of non-atomic interventions from observational and experimental distributions given a causal diagram.

\section{Preliminaries}

The basic semantic framework our work rests on is the Structural Causal Models paradigm (Pearl 2000, Ch. 7), which allows one to represent the data-generation process and different types of interventions:

Definition 1 (SCM). A Structural Causal Model $M$ is a 4tuple $\langle\mathbf{U}, \mathbf{V}, \mathcal{F}, P(\mathbf{u})\rangle$, where $\mathbf{U}$ is a set of exogenous (latent) variables; $\mathbf{V}$ is a set of endogenous (observed) variables; $\mathcal{F}$ is a collection of functions such that each variable $V_{i} \in \mathbf{V}$ is determined by a function $f_{i} \in \mathcal{F}$. Each $f_{i}$ is a mapping from a set of exogenous variables $U_{i} \subseteq \mathbf{U}$ and a set of endogenous variables $P a_{i} \subseteq \mathbf{V} \backslash\left\{V_{i}\right\}$ to the domain of $V_{i}$. The uncertainty is encoded through a probability distribution over the exogenous variables, $P(\mathbf{U})$.

Note that this definition allows for latent confounders, so the model is also known as Semi-Markovian. Each SCM $M$ is associated with a causal diagram where every $V_{i} \in \mathbf{V}$ is a vertex, there is a directed edge $\left(V_{j} \rightarrow V_{i}\right)$ for every $V_{i} \in \mathbf{V}$ and $V_{j} \in P a_{i}$, and a bidirected edge $\left(V_{i} \leftrightarrow V_{j}\right)$ for every pair $V_{i}, V_{j} \in \mathbf{V}$ such that $U_{i} \cap U_{j} \neq \emptyset\left(V_{i}\right.$ and $V_{j}$ have a common latent confounder).

We assume that the underlying model is recursive, that is, there are no cyclic dependencies among the variables. Equivalently, the causal diagram corresponding to the SCM is acyclic. The observable distribution is derived from $M$ as

$$
P(\mathbf{v})=\sum_{\mathbf{u}} \prod_{\left\{i \mid V_{i} \in \mathbf{v}\right\}} P\left(v_{i} \mid p a_{i}, u_{i}\right) P(\mathbf{u}),
$$

where every term $P\left(v_{i} \mid p a_{i}, u_{i}\right)$ is governed by the corresponding function $f_{i} \in \mathcal{F}$ that represents an autonomous mechanisms affecting only $V_{i}$, locally (Aldrich 1989). 
A $d o(\mathbf{X}=\mathbf{x})$ intervention results in a new structural causal model $M_{\mathbf{x}}$, which represents the state of the system after the hypothetical intervention takes place. As for $M$, assumptions about the causal structure of $M_{\mathbf{x}}$ can be seen as the corresponding causal diagram $\mathcal{G}_{\overline{\mathbf{X}}}$, which is the same as $\mathcal{G}$ but for the absence of all edges incoming towards $\mathbf{X}$. Moreover, $M_{\mathbf{x}}$ induces a probability distribution $P(\mathbf{V} \mid d o(\mathbf{x}))$ that can be established using Eq. (1) in the context of $M_{\mathbf{x}}$, i.e.:

$$
P(\mathbf{v} \mid \operatorname{do}(\mathbf{x}))=\sum_{\mathbf{u}} \prod_{\left\{i \mid V_{i} \in \mathbf{V}\right\}} P\left(v_{i} \mid p a_{i}, u_{i}, \operatorname{do}(\mathbf{x})\right) P(\mathbf{u} \mid \operatorname{do}(\mathbf{x})) .
$$

The key observation here is that for every $V_{i} \in \mathbf{V}, V_{i} \notin$ $\mathbf{X}, P\left(v_{i} \mid p a_{i}, u_{i}, d o(\mathbf{x})\right)=P\left(v_{i} \mid p a_{i}, u_{i}\right)$, because the functions $f_{i}$ in $M$ and $M_{\mathbf{x}}$ are the same. Similarly, $P(\mathbf{u} \mid d o(\mathbf{x}))=P(\mathbf{u})$ since exogenous variables are not affected by the do-operation. Moreover, for $V_{i} \in \mathbf{X}$, the function $f_{i}$ in $M_{\mathbf{x}}$ is independent of $\mathbf{U} \cup\left(\mathbf{V} \backslash\left\{V_{i}\right\}\right)$, hence $P a_{i}=\emptyset, U_{i}=\emptyset$, and the corresponding term $P\left(V_{i}=v_{i} \mid\right.$ $d o(\mathbf{x}))=1$, if $v_{i}$ is consistent with $\mathbf{x}$; and 0 , otherwise. Then, $P(\mathbf{v} \mid d o(\mathbf{x}))$ in Eq. (2) is also equal to:

$$
\left\{\begin{array}{ll}
\sum_{\mathbf{u}} \prod_{\left\{i \mid V_{i} \in \mathbf{V} \backslash \mathbf{X}\right\}} P\left(v_{i} \mid p a_{i}, u_{i}\right) P(\mathbf{u}) & \mathbf{v} \text { consistent with } \mathbf{x} \\
0 & \mathbf{v} \text { inconsistent with } \mathbf{x}
\end{array} .\right.
$$

In the special case of Markovian models, where every unobservable variable in $\mathrm{U}$ affects at most one observable, Eq. (3) is called the "truncated factorization product" (Pearl 1993a; 2000; Bareinboim, Brito, and Pearl 2012), which yields a mapping from the pre $(P(\mathbf{V}))$ to the post-interventional distribution $(P(\mathbf{V} \mid d o(\mathbf{x})))$. In Fig. 1(a), for example, the effect $P(y \mid d o(x))=\sum_{w, z} P(y \mid x, z, w) P(z \mid w) P(w)$ by Eq. (3).

It is unlikely that one could observe all variables in the system in most practical applications. Consequently, realistic causal diagrams usually account for latent (unobserved, exogenous) variables that affect more than one observable, which are represented through bidirected edges. In the following sections, we will address the problem of identifying the effect of stochastic interventions in such class of models.

We follow standard notation in the field. Random variables are denoted with uppercase letters (e.g, $C$ ) while their instantiations to particular values are written in lowercase (e.g, c). Similarly, letters in bold (e.g, C) represent sets of variables, and lowercase-bold letters (e.g., c) a particular value assignment for them. Further, we denote by $\mathcal{G}_{\overline{\mathbf{W}} \mathbf{x}}$ the graph that is the same as $\mathcal{G}$ except that the edges incoming to variables in $\mathbf{W}$ and the edges going out from variables in $\mathbf{X}$ are removed. Let $\mathcal{G}_{[\mathbf{C}]}$ be the subgraph of $\mathcal{G}$ made only of nodes in $\mathbf{C} \subset \mathbf{V}$ and the edges between them. We define $P a(\mathbf{C})$ and $A n(\mathbf{C})$, as the union of $\mathbf{C} \subset \mathbf{V}$ with its parents and ancestors, respectively. Also, the expression $(\mathbf{X} \Perp \mathbf{Y} \mid \mathbf{Z})_{\mathcal{G}}$ denotes that the variables in $\mathbf{X}$ are separated from the variables in $\mathbf{Y}$ conditioned on $\mathbf{Z}$ according to the d-separation criterion in the graph $\mathcal{G}$ (Pearl 2000).

The proofs are provided in the Appendix.

\section{Moving Beyond Atomic Interventions}

In general, the result of an intervention encompasses a new regime where the data-generating process differs from that of the natural system only in the mechanisms associated with the variables that have been intervened (Pearl 1994; Dawid 2002; 2015). From this point of view, we use regime indicators as discussed in (Pearl 2000, Sec. 3.2.2) and (Dawid 2002) to represent different types of interventions. The regime indicator for interventions on a variable $X$ is denoted by $\sigma_{X}$, and encodes the fact that the function $f_{x}$ in $M$ has been replaced by a new function $f_{x}^{*}$. This operation results in a new model $M_{\sigma_{X}}$, with causal diagram $\mathcal{G}_{\sigma_{X}}$, and inducing a distribution $P\left(\mathbf{V} ; \sigma_{X}\right)$. See Fig. 1(b)-(d) for a few examples of post-interventional diagrams.

In particular, depending on the intervention, the function $f_{x}^{*}$ could receive as inputs the values of variables other than the original parents $P a_{x}$ and $U_{x}$. Accordingly, we will denote as $P a_{x}^{*}$ and $U_{x}^{*}$ the set of observable and unobservable parents of $X$ in $M_{\sigma_{X}}$, as dictated by $f_{x}^{*}$. To avoid clutter, when a regime indicator $\sigma_{X}$ is present in a probability expression, such as $P\left(x \mid p a_{x}, u_{x} ; \sigma_{X}\right), P a_{x}$ and $U_{x}$ correspond to $P a_{x}^{*}$ and $U_{x}^{*}$, respectively. Naturally, this means that $\mathcal{G}_{\sigma_{X}}$ may not be a subgraph of $\mathcal{G}$, as it occurs with do-interventions. One important assumption used throughout the paper is that the hypothetical model $M_{\sigma_{X}}$ resulting from the intervention $\sigma_{X}$ does not contain cycles. Following the convention in (Dawid 2002), we augment $\mathcal{G}_{\sigma_{\mathbf{X}}}$ with a node $\sigma_{X_{i}}$ for every $X_{i} \in \mathbf{X}$ that graphically denote the targets of intervention, together with the edge $\left(\sigma_{X_{i}} \rightarrow X_{i}\right)$.

\section{Representing Different Types of Interventions}

Qualitatively different types of interventions can be modeled by assigning different strategies to the indicator $\sigma_{X}$ using the construct discussed above. We list in Table 1 general types of interventions that will be used in the remaining of the paper. The idle intervention represents the natural state of the system; atomic or do interventions replace the function $f_{X}$ with a constant, while conditional ones replace it with a deterministic function of some observables $p a_{x}^{*}$. The stochastic type sets the new $f_{X}^{*}$ such that the variable $X$ will follow a pre-specified distribution $P^{*}\left(X \mid p a_{x}^{*}\right)$. To simplify notation, whenever the strategy assigned to $\sigma_{X}$ is clear from the context, we will omit it in the probability expressions. Also, we may just write $P(\mathbf{V})$ whenever $P\left(\mathbf{V} ; \sigma_{X}=\emptyset\right)$. For a set $\mathbf{X} \subset \mathbf{V}$, let $\sigma_{\mathbf{X}}=\left\{\sigma_{X_{1}}, \ldots\right\}$ represent an intervention affecting the functions $f_{x_{i}}$ of every $X_{i} \in \mathbf{X}$.

Example 1 (Conditional Intervention). In the context of a tutoring program, suppose that in Fig. 1(a) $W$ represents previous GPA of a student, $Z$ student's motivation, $X$ after hours tutoring (or not), and $Y$ the GPA at the end of the term. Currently, students seek tutoring voluntarily, which depends on their motivation. Given the limited amount of resources, the school is considering to make after hours tutoring mandatory for students with low GPAs, and offering this service only to them. The proposed intervention can be encoded as $\sigma_{X}=g(w)$, where $g(w)=1$ if $W$ is low GPA, and 0 otherwise. Graphically, this change in policy is represented by the diagram in Fig. 1(c), where $X$ now depends on $W$, not on $Z$. Still, we highlight that $X$ was dependent on $Z$ in the observational regime and its corresponding dataset. 


\begin{tabular}{|c|c|c|c|}
\hline Type & Strategy & $P\left(x \mid p a_{x}, u_{x} ; \sigma_{X}\right)$ & \\
\hline Idle & $\emptyset$ & (unaltered) & \\
\hline Atomic/do & $d o\left(X=x^{\prime}\right)$ & $\delta\left(x, x^{\prime}\right)$ & (4) \\
\hline Conditional & $d o\left(X=g\left(p a_{x}^{*}\right)\right)$ & $\delta\left(x, g\left(p a_{x}^{*}\right)\right)$ & (5) \\
\hline Stochastic/Random & $P^{*}\left(X \mid p a_{x}^{*}\right)$ & $P^{*}\left(x \mid p a_{x}^{*}\right)$ & (6) \\
\hline
\end{tabular}

Table 1: (1st column) Different types of interventions. (2nd) The corresponding strategies that can be assigned to the indicator variable $\sigma_{X}$. (3rd) Distributions that $X$ will display after the intervention is implemented. $\delta(a, b)=1$ if $a=b$, and 0 otherwise.

Example 2 (Stochastic Intervention). Recall the discussion about the new smoking policy in the introduction. One could estimate the effect of reducing by $90 \%$ smoking on people under 21 years old by reasoning about a stochastic intervention $P^{*}(x \mid w, z)$, depicted in Fig.1(d), such that $P^{*}(X=1 \mid W<21, z)=(0.1) \times P(X=1 \mid z)$, for every $z$.

Interestingly, the randomization procedure used in a controlled experiment (Fisher 1951) - represented by the do-operator - can be seen as the implementation of the stochastic intervention $\sigma_{X}=P^{*}(X)$, with $P^{*}(x)=1 / 2$, for $x=\{0,1\}$. This procedure induces the distribution $P\left(\mathbf{v} ; \sigma_{X}=P^{*}(X)\right)$. Evidently, Fisher's randomization is physical, while the inferences studied here are about how to determine a causal effect without actually performing the intervention in the real world. To understand this connection more precisely, we first condition the postinterventional distribution, $P\left(\mathbf{v} ; \sigma_{X}=P^{*}(X)\right)$, on $X$, which leads to $P\left(\mathbf{v} \mid X=x ; \sigma_{X}=P^{*}(x)\right)$. Now notice that each individual for which $X=x$ under $\sigma_{X}$ is assigned treatment completely at random (i.e., without the influence of any other factor), which is the very definition of $d o(x)$, hence $P\left(\mathbf{v} \mid x ; \sigma_{X}=P^{*}(x)\right)=P(\mathbf{v} \mid d o(x))$.

\section{Effect of General Interventions}

Regardless of the particular type of intervention, we can reason about the distribution that (the hypothetical) $M_{\sigma_{\mathbf{X}}}$ induces. Let $\mathbf{U}^{*}$ be the set of all unobservable variables in $M_{\sigma_{\mathbf{X}}}$, then using Eq. (1) we have:

$$
P\left(\mathbf{v} ; \sigma_{\mathbf{X}}\right)=\sum_{\mathbf{u}^{*}} \prod_{\left\{i \mid V_{i} \in \mathbf{V}\right\}} P\left(v_{i} \mid p a_{i}, u_{i} ; \sigma_{X}\right) P\left(\mathbf{u}^{*} ; \sigma_{X}\right) .
$$

Every $V_{i} \in \mathbf{V} \backslash \mathbf{X}$ is governed by the same function in $M$ and $M_{\sigma_{X}}$, by definition, hence $P\left(v_{i} \mid p a_{i}, u_{i} ; \sigma_{\mathbf{X}}\right)=$ $P\left(v_{i} \mid p a_{i}, u_{i}\right)$. For the exogenous, the variables in the set $\mathbf{U}^{*} \backslash \mathbf{U}$ were introduced due to $\sigma_{\mathbf{X}}$ and were not originally in $M$ (e.g., the randomness for a stochastic intervention). Since $\mathbf{U}$ is not affected by $\sigma_{\mathbf{X}}$, it follows $P\left(\mathbf{u} ; \sigma_{\mathbf{X}}\right)=P(\mathbf{u})$, and

$$
\begin{gathered}
P\left(\mathbf{v} ; \sigma_{\mathbf{X}}\right)=\sum_{\mathbf{u}^{*}} \prod_{\left\{i \mid V_{i} \in \mathbf{X}\right\}} P\left(v_{i} \mid p a_{i}, u_{i} ; \sigma_{\mathbf{X}}\right) P\left(\mathbf{u}^{*} \backslash \mathbf{u} ; \sigma_{\mathbf{X}}\right) \\
\prod_{\left\{i \mid V_{i} \in \mathbf{V} \backslash \mathbf{X}\right\}} P\left(v_{i} \mid p a_{i}, u_{i}\right) P(\mathbf{u}) .
\end{gathered}
$$

While Eq. (8) holds in general, the distribution $P(\mathbf{U})$ is not observed. The challenge is then to find a function of the observed distribution $P(\mathbf{V})$ that is guaranteed to be equal to the probability query of interest in the intervened model $M_{\sigma_{\mathbf{X}}}$, for any $M$ inducing $\mathcal{G}$. Formally,
Definition 2 (Effect Identifiability). Let $\mathbf{Y}, \mathbf{X}, \mathbf{W} \subset \mathbf{V}$ with $\mathbf{W} \cap \mathbf{Y}=\emptyset$. The (conditional) effect of an intervention specified by $\sigma_{\mathbf{X}}=\left\{\sigma_{X_{1}}, \ldots, \sigma_{X_{n}}\right\}$ on a set of outcome variables $\mathbf{Y}$, conditional on $\mathbf{W}, P\left(\mathbf{y} \mid \mathbf{w} ; \sigma_{\mathbf{X}}\right)$, is said to be identifiable in $\mathcal{G}$, if it is uniquely computable from the joint distribution $P(\mathbf{V})$, for every assignment $(\mathbf{y}, \mathbf{w})$, in every model that induces $\mathcal{G}$ and $P(\mathbf{V})$.

Remark 1. An important distinction between atomic and more general interventions is that the former implicitly conditions on the intervened variable $\mathbf{X}$, more formally,

$$
\begin{aligned}
P(\mathbf{y} \mid \operatorname{do}(\mathbf{x})) & =P\left(\mathbf{y} ; \sigma_{\mathbf{X}}=d o(\mathbf{X}=\mathbf{x})\right) \\
& =P\left(\mathbf{y} \mid \mathbf{x} ; \sigma_{\mathbf{X}}=d o(\mathbf{X}=\mathbf{x})\right) .
\end{aligned}
$$

Eq. (9) follows by definition, and Eq. (10) is immediate since under the intervention $\sigma_{X}=d o(\mathbf{X}=\mathbf{x})$, the probability of $\mathbf{X}$ being different than the constant $\mathbf{x}$ is zero. In general, $P\left(\mathbf{y} ; \sigma_{\mathbf{X}}\right)$ and $P\left(\mathbf{y} \mid \mathbf{x} ; \sigma_{\mathbf{X}}\right)$ need not match one another.

Interestingly, while atomic interventions always reduce the model structure, a policy-maker could envision a new policy taking into account a wide range of covariates, not matching the observational regime and previous policies (as with Examples 1, 2).

\section{A Calculus for General Interventions}

In this section, we introduce a set of inference rules, in the spirit of do-calculus (Pearl 2000, Sec. 3.4), capable of handling both atomic and non-atomic interventions, which we call $\sigma$-calculus.

Theorem 1. [Inference Rules - $\sigma$-calculus] Let $\mathcal{G}$ be a causal diagram compatible with a structural causal model $M$, with endogenous variables $\mathbf{V}$. For any disjoint subsets $\mathbf{X}, \mathbf{Y}, \mathbf{Z} \subseteq \mathbf{V}$, two disjoint subsets $\mathbf{T}, \mathbf{W} \subseteq \mathbf{V} \backslash(\mathbf{Z} \cup \mathbf{Y})$ (i.e., possibly including $\mathbf{X}$ ), the following rules are valid for any intervention strategies $\sigma_{\mathbf{X}}, \sigma_{\mathbf{Z}}$, and $\sigma_{\mathbf{Z}}^{\prime}$ :

Rule 1 (Insertion/Deletion of observations):

$$
\begin{gathered}
P\left(\mathbf{y} \mid \mathbf{w}, \mathbf{t} ; \sigma_{\mathbf{X}}\right)=P\left(\mathbf{y} \mid \mathbf{w} ; \sigma_{\mathbf{X}}\right) \\
\text { if }(\mathbf{Y} \Perp \mathbf{T} \mid \mathbf{W}) \text { in } \mathcal{G}_{\sigma_{\mathbf{X}}} .
\end{gathered}
$$

Rule 2 (Change of regimes under observation):

$$
\begin{aligned}
& P\left(\mathbf{y} \mid \mathbf{z}, \mathbf{w} ; \sigma_{\mathbf{x}}, \sigma_{\mathbf{z}}\right)=P\left(\mathbf{y} \mid \mathbf{z}, \mathbf{w} ; \sigma_{\mathbf{x}}, \sigma_{\mathbf{z}}^{\prime}\right) \\
& \quad \text { if }(\mathbf{Y} \Perp \mathbf{Z} \mid \mathbf{W}) \text { in } \mathcal{G}_{\sigma_{\mathbf{X}} \sigma_{\mathbf{z}} \underline{\mathbf{z}}} \text { and } \mathcal{G}_{\sigma_{\mathbf{X}} \sigma_{\mathbf{z}}^{\prime} \underline{\mathbf{z}}} .
\end{aligned}
$$

Rule 3 (Change of regimes without observation):

$$
\begin{aligned}
& P\left(\mathbf{y} \mid \mathbf{w} ; \sigma_{\mathbf{x}}, \sigma_{\mathbf{z}}\right)=P\left(\mathbf{y} \mid \mathbf{w} ; \sigma_{\mathbf{x}}, \sigma_{\mathbf{z}}^{\prime}\right) \\
& \text { if }(\mathbf{Y} \Perp \mathbf{Z} \mid \mathbf{W}) \text { in } \mathcal{G}_{\sigma_{\mathbf{X}} \sigma_{\mathbf{Z}} \overline{\mathbf{Z}(\mathbf{W})}} \text { and } \mathcal{G}_{\sigma_{\mathbf{X}} \sigma_{\mathbf{Z}}^{\prime}} \overline{\mathbf{Z}(\mathbf{W})} \text {, }
\end{aligned}
$$


where $\mathbf{Z}(\mathbf{W}) \subseteq \mathbf{Z}$ is the set of elements in $\mathbf{Z}$ that are not ancestors of $\mathbf{W}$ in $\mathcal{G}_{\sigma_{\mathbf{X}}}$.

The rules above follow from the semantics of $\sigma_{\mathbf{X}}=$ $\left\{\sigma_{X_{1}}, \ldots, \sigma_{X_{k}}\right\}$ as indicator of the change in causal mechanism of each variable in $\mathbf{X}$ according to a specified strategy. Rule 1 ascertains the validity of the $d$-separation criterion for reading conditional independence constraints in the post-interventional distribution $P\left(\mathbf{V} ; \sigma_{\mathbf{X}}\right)$ using the interventional graph $\mathcal{G}_{\sigma_{\mathbf{X}}}$. Rule 2 establishes a condition that guarantees that the corresponding probability distribution is the same under interventions $\sigma_{\mathbf{Z}}^{\prime}$ and $\sigma_{\mathbf{Z}}$ while $\mathbf{Z}=\mathbf{z}$ is observed. Rule 3 establishes a condition for changing the regime indicator from $\sigma_{\mathbf{Z}}^{\prime}$ to $\sigma_{\mathbf{Z}}$ without affecting the associated probability. This rule differs from rule 2 since it is only applicable when $\mathbf{Z}$ is not observed.

In particular, these rules can be applied with $\sigma_{\mathbf{Z}}^{\prime}$ having $\sigma_{Z_{i}}=\emptyset$, to make one or more regime indicators for $Z_{i} \in \mathbf{Z}$ idle. When all indicators are idle the expression is estimable from observational data. Differently than in the case of atomic interventions and $d o$-calculus, causal diagrams induced by intervened models in this context are not necessarily subgraphs of the original diagram, hence $\sigma$-calculus needs to verify separation conditions in the corresponding two models. In Appendix. B, we revisit a classical example from (Pearl and Robins 1995) that misses this subtlety and reaches an incorrect conclusion. The same appendix also provides a more detailed comparison of both calculi.

\section{Comparison between $\sigma$-calculus and $d o$-calculus}

Independences in do-calculus rules usually include conditioning on $\mathbf{X}$. Notice that in our rules $\mathbf{W}$ could include variables in $\mathbf{X}$, accounting for situations when the expression has conditioning on $\mathbf{X}$ or part of it, but not necessarily the whole set every time.

The new rule 2 allows one to change across regimes when the variable under intervention is being observed. This is consistent with the traditional rule 2 and remark 1 about the $d o($.$) operator having an implicit conditioning on the inter-$ vened variable. Consider the back-door graph in Fig. 1(a) and an intervention $\sigma_{X}=d o(g(z))$, which is associated with $\mathcal{G}_{\sigma_{X}}=\mathcal{G}$ (same argument in the observational and new interventional regime). Using the new rule 2, we have:

$$
P\left(y \mid x, z ; \sigma_{X}\right)=P(y \mid x, z),
$$

since $(X \Perp Y \mid Z)$ holds in both $\mathcal{G}_{\underline{X}}$ and $\mathcal{G}_{\sigma_{X} \underline{X}}$ (same graph in this case, Fig. 2(a)). One may be tempted to apply the new rule 2 as its $d o$-calculus counterpart, trying to claim that

$$
P\left(y \mid z ; \sigma_{X}\right)=P(y \mid x, z) .
$$

However, this is not the case for many models compatible with the graph (see appendix for details).

Rule 3 licenses the addition or removal of a regime altogether. This rule is not the exact counterpart of the same rule in do-calculus. Consider again Fig. 1(a) with $\sigma_{Z}=P^{*}(z \mid w)$ and its effect on $W$ conditioned on $Z$. Traditional rule 3 tests for $(W \Perp Z)$ in $\mathcal{G}_{\bar{Z}}$ which leads to

$$
P(w \mid d o(z))=P\left(w \mid z ; \sigma_{Z}\right)=P(w) .
$$

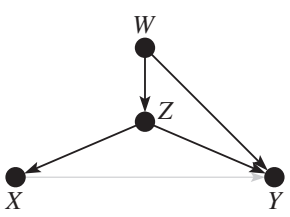

(a) $\mathcal{G}_{\underline{X}}$

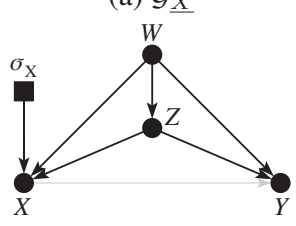

(c) $\mathcal{G}_{\sigma_{X} \underline{X}}$

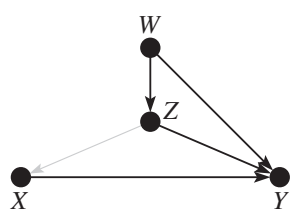

(b) $\mathcal{G}_{\bar{X}}$

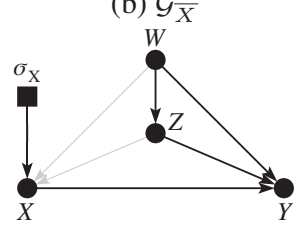

(d) $\mathcal{G}_{\sigma_{X} \bar{X}}$
Figure 2: Graphs used to test the conditions required by rules 2 and 3 of $\sigma$-calculus in the derivation of the query in Example 2 where $\sigma_{X}=P^{*}(x \mid z, w)$. Arrows shown in gray indicate they have been cut.

In contrast, we consider the $\sigma_{Z}$ in $\sigma$-calculus:

$$
P\left(w \mid z ; \sigma_{Z}\right)=P(w) P\left(z \mid w ; \sigma_{Z}\right) / P\left(z ; \sigma_{Z}\right),
$$

which is almost always different than $P(w)$. The important distinction to make at this point is that for soft intervention on $Z$, we are not necessarily conditioning on it. Without conditioning, rule 3 of $\sigma$-calculus and independence $(W \Perp Z)$ in $\mathcal{G}_{\bar{Z}}$ (and $\mathcal{G}_{\sigma_{Z}} \bar{Z}$ ) yield $P\left(w ; \sigma_{Z}\right)=P(w)$. In contrast

$$
P\left(w \mid z ; \sigma_{Z}=d o(z)\right)=P\left(w ; \sigma_{Z}=d o(z)\right)=P(w)
$$

can be obtained by applying first rule 1 with $(W \Perp Z)$ in $\mathcal{G}_{\sigma_{Z}=d o(z)}$ and then rule 3 (more discussion in appendix).

\section{Examples of Symbolic Derivations}

We illustrate the use of $\sigma$-calculus rules by solving the question in Example 2. Recall that our goal is to identify $P\left(y ; \sigma_{X}\right)$ with $\sigma_{X}=P^{*}(x \mid w, z)$. We start by conditioning on the set $\{X, Z, W\}$,

$$
\begin{aligned}
& P\left(y ; \sigma_{X}\right)= \\
& \sum_{x, z, w} P\left(y \mid x, z, w ; \sigma_{X}\right) P\left(x \mid z, w ; \sigma_{X}\right) P\left(z, w ; \sigma_{X}\right),
\end{aligned}
$$

Note that Rule 2 can be applied with $\sigma_{X}^{\prime}=\emptyset$ to infer $P\left(y \mid x, z, w ; \sigma_{X}\right)=P(y \mid x, z, w)$ following the independence $(Y \Perp X \quad Z, W)$ in the $\mathcal{G}_{X}$ and $\mathcal{G}_{\sigma_{X} X}$ (see Figs. 2(a) and (c), respectively). Also, Rule $3\left(\sigma_{X}^{\prime}=\emptyset\right)$ leads to $P\left(z, w ; \sigma_{X}\right)=P(z, w)$, licensed by $(Z, W \Perp X)$ in $\mathcal{G}_{\bar{X}}$ and $\mathcal{G}_{\sigma_{X}} \bar{X}$ (Figs. 2(b), (d)). Next, we replace $P\left(x \mid z, w ; \sigma_{X}\right)$ using Eq. (6) by virtue of $\sigma_{X}=P^{*}(x \mid z, w)$ :

$$
P\left(y ; \sigma_{X}\right)=\sum_{x, z, w} P(y \mid x, z, w) P^{*}(x \mid z, w) P(z, w) .
$$

Notice that all terms in the right hand side of Eq. (15) are either obtainable from $P(\mathbf{V})$ or defined by the new intervention itself, which means the target effect is identifiable (see appendix B for a more detailed example).

A natural albeit important consequence of Thm. 1 is described in the following corollary:

Corollary 1. Considering only atomic (and idle) interventions, $\sigma$-calculus reduces to do-calculus. 


\section{Identifying Effects with (Atomic and Non-atomic) Surrogate Experiments}

It's not uncommon that the effect of a certain intervention is not identifiable from observational data alone whenever unobserved confounders are present. It may be the case that experiments over surrogate variables may be available for use, which has been called in the literature the problem of z-identification (Bareinboim and Pearl 2012; Lee, Correa, and Bareinboim 2019). For instance, experiments over a set of surrogate variables $\mathbf{Z}$ may be more accessible to manipulation than the target effect $\sigma_{\mathbf{X}}$. In this case, still, experiments are assumed to be the product of controlled trials, that is, of atomic interventions. In this section we leverage data from surrogate experiments obtained from general intervention.

Example 3. To illustrate, consider the causal diagram in Fig. 3(a) and the effect $P\left(y \mid r, z ; \sigma_{X}=P^{*}(X \mid R)\right)$, which is not identifiable from $P(\mathbf{V})$. However, suppose a distribution $P\left(\mathbf{V} ; \sigma_{Z}=P^{*}(Z \mid X)\right)$ is given as an additional input. We can then write

$$
\begin{aligned}
& P\left(y \mid r, z ; \sigma_{X}\right) \\
& =P\left(y \mid r, z ; \sigma_{X}, \sigma_{Z}=d o(z)\right) \\
& =P\left(y \mid r, z ; \sigma_{Z}=d o(z)\right) \\
& =\sum_{x^{\prime}} P\left(y \mid r, x^{\prime}, z ; \sigma_{Z}=d o(z)\right) P\left(x^{\prime} \mid r, z ; \sigma_{z}=d o(z)\right) .
\end{aligned}
$$

Eq. (16) follows from Rule 2 and the independence $(Y \Perp$ $Z \mid R)$ in $\mathcal{G}_{\sigma_{X} \sigma_{Z}=\emptyset \underline{Z}}$ and $\mathcal{G}_{\sigma_{X} \sigma_{Z}=d o(z) \underline{Z}}$; Eq. (17) by Rule 3 with $(Y \Perp \bar{X} \mid R, Z)$ in $\mathcal{G}_{\sigma_{X} \sigma_{Z}=d o(z) \bar{X}}$ and $\mathcal{G}_{\sigma_{X}=\emptyset \sigma_{Z}=\operatorname{do}(z) \bar{X}}$. At this point, note that if the given experiment was randomized (i.e., $d o(z)$ ), the target effect would be identifiable. However, the given distribution came from policy $\sigma_{Z}=P^{*}(Z \mid X)$. Nevertheless, we can condition on $X$ and obtain Eq. (18). We can then apply Rule 2 to change the strategy of $\sigma_{Z}$ from $d o(Z)$ to $P^{*}(Z \mid X)$ due to $(Y \Perp$ $Z \mid R, X)$ in $\mathcal{G}_{\sigma_{Z}=d o(z) \underline{Z}}$ and $\mathcal{G}_{\sigma_{Z}=P^{*}(Z \mid X) \underline{Z}}$ that license $P\left(y \mid r, x^{\prime}, z ; \sigma_{Z}=d o(z)\right)=P\left(y \mid r, x^{\prime}, z ; \sigma_{Z}=P^{*}(Z \mid X)\right)$. Finally, the second factor in Eq. (18) can be obtained from the observational data by applying Rule 1 with $(X \Perp Z)$ in $\mathcal{G}_{\sigma_{Z}=d o(z)}$ to remove the observation on $z$, followed by Rule 3 and $(X \Perp Z \mid R)$ in $\mathcal{G}_{\sigma_{Z} \bar{Z}}$ and $\mathcal{G}_{\sigma_{Z}=\emptyset \bar{Z}}$ to change $\sigma_{Z}$ to the idle regime. Putting the pieces together, we obtain the following expression:

$$
P\left(y \mid r, z ; \sigma_{X}\right)=\sum_{x^{\prime}} P\left(y \mid r, x^{\prime}, z ; \sigma_{Z}=P^{*}(Z \mid X)\right) P\left(x^{\prime} \mid r\right) .
$$

\section{Identifying the Effect of General Interventions Systematically}

Even though $\sigma$-calculus is a great tool for understanding and reasoning about the logical implications of general interventions, searching for a derivation in moderately-sized causal models can be a very challenging task given the combinatorial nature of the problem. Also, the solution of realistic applications involving models with thousands of variables requires the use of computers. In this section, we develop an

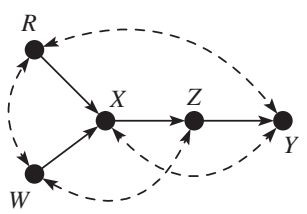

(a) $\mathcal{G}$

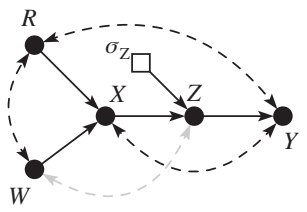

(c) $\mathcal{G}_{\sigma_{Z}=P^{*}(Z \mid X)}$

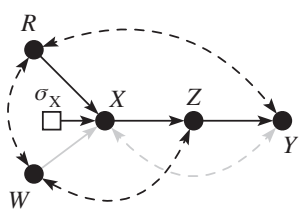

(b) $\mathcal{G}_{\sigma_{X}}$

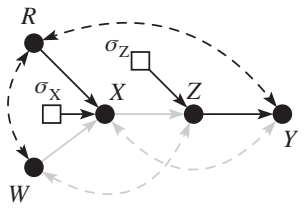

(d) $\mathcal{G}_{\sigma_{X} \sigma_{Z}}=\operatorname{do}(z)$
Figure 3: (a) is the original diagram for which we want to identify the effect $P\left(y \mid r, z ; \sigma_{X}=P^{*}(X \mid R)\right)$ corresponding to the diagram in (b). Experimental data is given in the form of $P\left(\mathbf{V} ; \sigma_{Z}=P^{*}(Z \mid X)\right)$ corresponding to (c). Diagram in (d) is intermediate in the derivation of the target effect (see text for details).

algorithmic solution for identifying the (conditional) effect of general interventions (Table 1) from observational and experimental data, based on a given causal diagram $\mathcal{G}$.

Consider a query of interest $P\left(\mathbf{y}, \mathbf{w} ; \sigma_{\mathbf{X}}\right)$ and let $\mathbf{D}=$ $A n(\mathbf{Y} \cup \mathbf{W})_{\mathcal{G}_{\sigma_{\mathbf{X}}}}$, then from Eq. (7) we can sum out variables that are not ancestors of $(\mathbf{Y} \cup \mathbf{W})$ and obtain

$$
\begin{aligned}
& P\left(\mathbf{y}, \mathbf{w} ; \sigma_{\mathbf{X}}\right)=\sum_{\mathbf{v} \backslash(\mathbf{y} \cup \mathbf{w})} P\left(\mathbf{v} ; \sigma_{\mathbf{X}}\right) \\
& \quad=\sum_{\mathbf{d} \backslash(\mathbf{y} \cup \mathbf{w})} \sum_{\mathbf{u}^{*}} \prod_{\left\{i \mid V_{i} \in \mathbf{D}\right\}} P\left(v_{i} \mid p a_{i}, u_{i} ; \sigma_{\mathbf{X}}\right) P\left(\mathbf{u}^{*} ; \sigma_{\mathbf{X}}\right) .
\end{aligned}
$$

For convenience, and following (Tian and Pearl 2002a), we define for any $\mathbf{C} \subseteq \mathbf{V}$ the quantity $Q[\mathbf{C}](\mathbf{v})$, called $c$ factor, to denote the following function

$$
\begin{aligned}
& Q\left[\mathbf{C} ; \sigma_{\mathbf{X}}\right](\mathbf{v})= \\
& \sum_{u(\mathbf{C})} \prod_{\left\{i \mid V_{i} \in \mathbf{C}\right\}} P\left(v_{i} \mid p a_{i}, u_{i} ; \sigma_{\mathbf{X}}\right) P\left(u(\mathbf{C}) ; \sigma_{\mathbf{X}}\right),
\end{aligned}
$$

where $U(\mathbf{C})=\bigcup_{V_{i} \in \mathbf{C}} U_{i}$. In particular, note that $Q\left[\mathbf{V} ; \sigma_{\mathbf{X}}\right](\mathbf{v})=P\left(\mathbf{v} ; \sigma_{\mathbf{X}}\right)$ and when $\sigma_{\mathbf{X}}=\emptyset, Q\left[\mathbf{C} ; \sigma_{\mathbf{X}}\right]=$ $Q[\mathbf{C}]$. For convenience, we will often write $Q[\mathbf{C}](\mathbf{v})$ as $Q[\mathbf{C}]$, and whenever $\mathbf{C}=\left\{V_{i}\right\}$ we will write $Q\left[V_{i}\right]$ instead of $Q\left[\left\{V_{i}\right\}\right]$. Using c-factors, Eq. (20) translates into $P\left(\mathbf{y}, \mathbf{w} ; \sigma_{\mathbf{X}}\right)=\sum_{\mathbf{d} \backslash(\mathbf{y} \cup \mathbf{w})} Q\left[\mathbf{D} ; \sigma_{\mathbf{X}}\right]$. Now consider the query $P\left(\mathbf{y} \mid \mathbf{w} ; \sigma_{\mathbf{X}}\right)$, we can write

$$
P\left(\mathbf{y} \mid \mathbf{w} ; \sigma_{\mathbf{X}}\right)=\frac{P\left(\mathbf{y}, \mathbf{w} ; \sigma_{\mathbf{X}}\right)}{\sum_{\mathbf{y}} P\left(\mathbf{y}, \mathbf{w} ; \sigma_{\mathbf{X}}\right)}=\frac{\sum_{\mathbf{d} \backslash(\mathbf{y} \cup \mathbf{w})} Q\left[\mathbf{D} ; \sigma_{\mathbf{X}}\right]}{\sum_{\mathbf{d} \backslash \mathbf{w}} Q\left[\mathbf{D} ; \sigma_{\mathbf{X}}\right]} .
$$

Moreover, Eq. (22) can be further simplified as stated in the following

Lemma 1. Let $\mathbf{Y}, \mathbf{X}, \mathbf{W} \subset \mathbf{V}$ with $\mathbf{W} \cap \mathbf{Y}=\emptyset$ and let $\mathcal{G}$ be a causal diagram over the variables $\mathbf{V}$. The effect $P(\mathbf{y}$ $\left.\mathbf{w} ; \sigma_{\mathbf{X}}\right)$ is given by:

$$
P\left(\mathbf{y} \mid \mathbf{w} ; \sigma_{\mathbf{X}}\right)=\sum_{\mathbf{a} \backslash(\mathbf{y} \cup \mathbf{w})} Q\left[\mathbf{A} ; \sigma_{\mathbf{X}}\right] / \sum_{\mathbf{a} \backslash \mathbf{w}} Q\left[\mathbf{A} ; \sigma_{\mathbf{X}}\right],
$$


where $\mathbf{A}$ is the set of all variables connected to $\mathbf{Y}$ (including $\mathbf{Y}$ ) by any path (regardless of the directionality) in

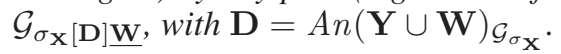

The problem we need to solve now is to determine if, and how, the c-factor $Q\left[\mathbf{A} ; \sigma_{\mathbf{X}}\right]$ can be computed from the observed data (i.e., $P(\mathbf{V})=Q[\mathbf{V}]$ ). To do so, we will leverage the machinery developed by (Tian and Pearl 2002a; Huang and Valtorta 2006) that deals with the identification of c-factors from other (larger) c-factors. First, note that the set of observable variables present in a causal diagram $\mathcal{G}$ can be partitioned into sets called c-components (Tian and Pearl 2002a). Two variables are in the same c-component set if and only if they are connected by a path composed entirely of bidirected edges in $\mathcal{G}$. Using this notion we state the following results, which will be key for our algorithm:

Lemma 2. Let $\mathbf{A}$ be defined relative to $\mathbf{X}, \mathbf{Y}, \mathbf{W}$ as in lemma 1, then:

$$
Q\left[\mathbf{A} ; \sigma_{\mathbf{X}}\right]=Q\left[\mathbf{A}^{\mathbf{x}} ; \sigma_{\mathbf{X}}\right] Q\left[\mathbf{A} \backslash \mathbf{A}^{\mathbf{X}}\right],
$$

where $\mathbf{A}^{\mathbf{X}}$ is the union of the c-components of $\mathcal{G}_{\sigma_{\mathbf{X}}[\mathbf{A}]}$ containing variables in $\mathbf{X}$.

Note that the factor $Q\left[\mathbf{A} \backslash \mathbf{A}^{\mathbf{X}}\right]$ in Eq. (24) corresponds to the idle regime. Hence, to asses if such c-factor is computable from $P(\mathbf{V})$ or a given $P\left(\mathbf{V} ; \sigma_{\mathbf{Z}}\right)$, we can use the algorithm IDENTIFY from (Tian and Pearl 2002a) and the following lemma.

Lemma 3. Let $\sigma_{\mathbf{Z}}$ indicate any intervention on $\mathbf{Z}$ and let $\mathbf{C} \subseteq \mathbf{V}$. Then, $Q[\mathbf{C}]=Q\left[\mathbf{C} ; \sigma_{\mathbf{Z}}\right]$ if $\mathbf{C} \cap \mathbf{Z}=\emptyset$.

What is left is to reason about the c-factor $Q\left[\mathbf{A}^{\mathbf{X}} ; \sigma_{\mathbf{X}}\right]$. In the case of atomic, conditional and stochastic interventions; $\mathbf{A}^{\mathbf{X}}$ is simply $\mathbf{X}$ because for those interventions, variables in $\mathbf{X}$ do not share unobservable parents with any other variable under intervention. Therefore, $Q\left[\mathbf{A}^{\mathbf{X}} ; \sigma_{\mathbf{X}}\right]=$ $\prod_{X \in \mathbf{X}} Q\left[X ; \sigma_{\mathbf{X}}\right]$ where each $Q\left[X ; \sigma_{\mathbf{X}}\right]=Q\left[X ; \sigma_{X}\right]$ is obtained by replacing it with the corresponding equation among (4), (5) or (6).

Following the discussion in this section, we propose the algorithm $\sigma$-IDENTIFY (Alg. 1). This procedure takes as input the variables defining a query, the specification of $\sigma_{\mathbf{X}}$ (i.e., what type of intervention is being applied and its arguments), a set of available distributions ( $\mathbb{Z}=\left\{\sigma_{\emptyset}\right\}$ when only $P(\mathbf{V})$ is known.) and the causal diagram. The subroutine 'REPLACE' handles factors of intervened variables, replacing them according to the type of intervention. $\sigma$-IDENTIFY runs in $O\left(n^{4} z\right)$ time, where $n$ is the number of nodes in $\mathcal{G}$ and $z=|\mathbb{Z}|$ (see Appendix C).

For an illustration, we run $\sigma$-IDENTIFY to identify $P\left(y \mid r ; \sigma_{X}=P^{*}(X \mid R)\right)$ in Fig. 3(a), where $\mathbf{Y}=\{Y\}$, $\mathbf{W}=\{R\}$ and $\sigma_{\mathbf{X}}=\left\{\sigma_{X}=P^{*}(X \mid R)\right\}$; from observation and experimental data $\mathbb{Z}=\left\{\sigma_{\emptyset}, \sigma_{Z}=P^{*}(Z \mid X)\right\}$. Here, $\mathbf{A}=\{R, X, Z, Y\}$ and the c-components of $\mathcal{G}_{\sigma_{X}[\mathbf{A}]}$ are $\mathbf{A}_{1}=\{R, Y\}, \mathbf{A}_{2}=\{Z\}$ and $\mathbf{A}_{3}=\{X\}$. The loop in line 2 will pick up $\mathbf{A}_{1}$ with $\sigma_{\mathbf{Z}}=\sigma_{Z}=P^{*}(Z \mid X)$, where $\mathbf{B}_{i}=\{R, X, Y, W\}$ is the c-component of $\mathcal{G}_{\sigma_{\mathbf{Z}}}$ (Fig. 3(c)) containing $\mathbf{A}_{1}$, for which IDENTIFY will return $\sum_{x^{\prime}} P\left(y \mid r, x^{\prime}, z ; \sigma_{Z}\right) P\left(x^{\prime}, r ; \sigma_{Z}\right)$. Next, in the same loop, $\mathbf{A}_{2}$ matches with $\sigma_{\mathbf{Z}}=\sigma_{\emptyset}$ and IDENTIFY returns

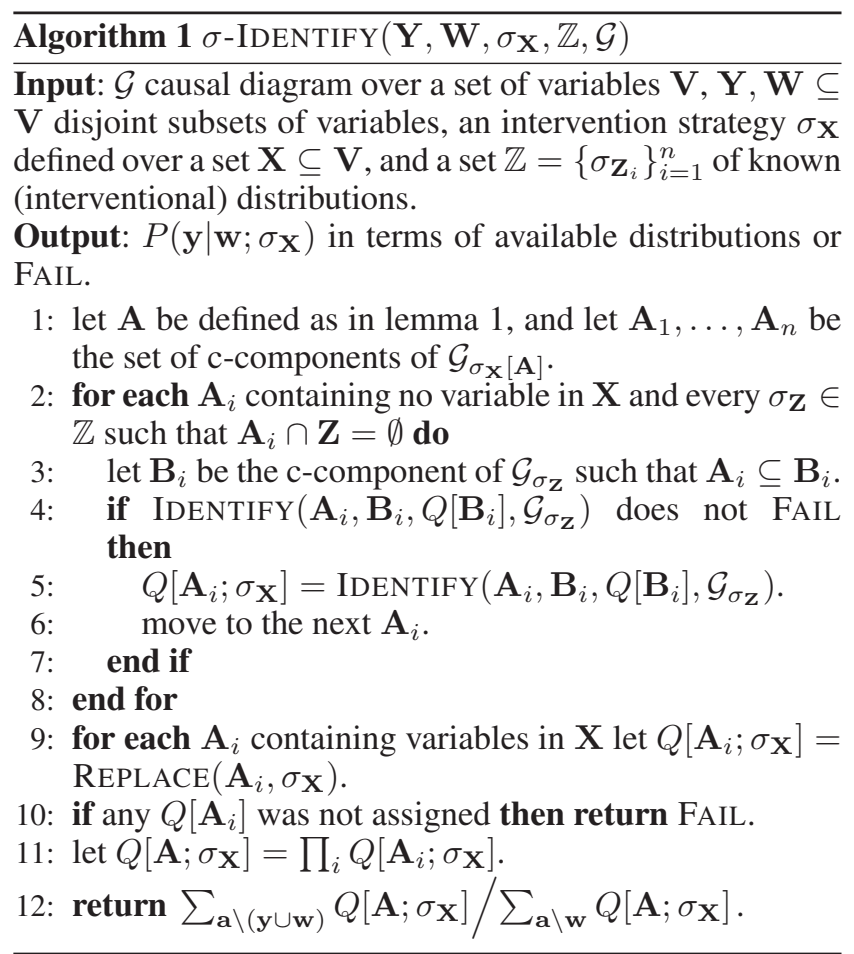

$\sum_{r^{\prime}, w} P\left(z \mid r^{\prime}, w, x\right) P\left(r^{\prime}, w\right)$. Line 9 handles $\mathbf{A}_{3}$ and replaces it with $P^{*}(x \mid r)$ according to the intervention $\sigma_{\mathbf{X}}$. Finally, the return expression is

$$
P\left(y \mid r ; \sigma_{X}\right)=Q\left[\mathbf{A} ; \sigma_{\mathbf{X}}\right] / \sum_{y} Q\left[\mathbf{A} ; \sigma_{\mathbf{X}}\right],
$$

where

$$
\begin{array}{r}
Q\left[\mathbf{A} ; \sigma_{\mathbf{X}}\right]=\sum_{x, z} P^{*}(x \mid r)\left(\sum_{x^{\prime}} P\left(y \mid r, x^{\prime}, z ; \sigma_{Z}\right) P\left(r, x^{\prime} ; \sigma_{Z}\right)\right) \\
\left(\sum_{r^{\prime}, w} P\left(z \mid r^{\prime}, w, x\right) P\left(r^{\prime}, w\right)\right)
\end{array}
$$

Theorem 2. The effect $P\left(\mathbf{y} \mid \mathbf{w} ; \sigma_{\mathbf{X}}\right)$ is identifiable if $\sigma$ IDENTIFY (Alg. 1) does not fail. Moreover, the expression returned is a valid estimand for the effect.

\section{Conclusions}

In this paper, we introduced a set of inference rules for reasoning about the effect of general interventions (Thm. 1), which has been called $\sigma$-calculus. The $\sigma$-calculus allows one to discover and verify from the causal graph, logical statements about general interventions generated by an arbitrary SCM. We showed how these rules can be used to identify the effect of interventions from a combination of observational and experimental data. Finally, we developed an algorithm (Alg. 1) that decides in an automated fashion whether a reduction of the effect of interest to the set of observed quantities (observational and experimental) exists; if so, it also returns the corresponding mapping. The algorithm and $\sigma$-calculus were proven sound and efficient for the task of identification of general interventions (Thm. 2), subsuming previous treatment for atomic interventions by do-calculus. 


\section{Acknowledgements}

This research was supported by grants from IBM Research, Adobe Research, NSF IIS-1704352, and IIS-1750807 (CAREER).

\section{References}

Aldrich, J. 1989. Autonomy. Oxford Economic Papers 41:15-34. Bareinboim, E., and Pearl, J. 2012. Causal Inference by Surrogate Experiments: z-Identifiability. In Proceedings of the TwentyEighth Conference on Uncertainty in Artificial Intelligence, 113120. AUAI Press.

Bareinboim, E., and Pearl, J. 2014. Transportability from Multiple Environments with Limited Experiments: Completeness Results. In Advances in Neural Information Processing Systems 27, 280 288.

Bareinboim, E., and Pearl, J. 2016. Causal inference and the datafusion problem. Proceedings of the National Academy of Sciences 113(27):7345-7352.

Bareinboim, E.; Brito, C.; and Pearl, J. 2012. Local Characterizations of Causal Bayesian Networks. In Croitoru, M.; Rudolph, S.; Wilson, N.; Howse, J.; and Corby, O., eds., GKR 2011, LNAI 7205, $1-17$.

Cartwright, N. 2007. Hunting Causes and Using Them: Approaches in Philosophy and Economics. New York, NY: Cambridge University Press.

Correa, J. D., and Bareinboim, E. 2019. From Statistical Transportability to Estimating the Effects of Stochastic Interventions. In Proceedings of the 27th International Joint Conference on Artificial Intelligence.

Dawid, A. P. 2002. Influence diagrams for causal modelling and inference. International Statistical Review 70:161-189.

Dawid, A. P. 2015. Statistical Causality from a Decision-Theoretic Perspective. Annual Review of Statistics and Its Application 2(1):273-303.

Didelez, V.; Dawid, A. P.; and Geneletti, S. 2006. Direct and indirect effects of sequential treatments. In Proceedings of the TwentySecond Conference on Uncertainty in Artificial Intelligence, 138146. AUAI Press.

Fisher, R. A. 1951. The Design of Experiments. Edinburgh: Oliver and Boyd, 6th edition.

Haavelmo, T. 1943. The Statistical Implications of a System of Simultaneous Equations. Econometrica 11(1):1-12.

Heckman, J. J. 2005. The Scientific Model of Causality. Sociological Methodology 35:1-97.

Huang, Y., and Valtorta, M. 2006. Identifiability in Causal Bayesian Networks: A Sound and Complete Algorithm. In Proceedings of the Twenty-First National Conference on Artificial Intelligence (AAAI 2006). AAAI Press. 1149-1156.

Huang, Y., and Valtorta, M. 2008. On the completeness of an identifiability algorithm for semi-Markovian models. Annals of Mathematics and Artificial Intelligence 54(4):363-408.

Lee, S.; Correa, J. D.; and Bareinboim, E. 2019. General Identifiability with Arbitrary Surrogate Experiments. In Proceedings of the Thirty-Fifth Conference Annual Conference on Uncertainty in Artificial Intelligence. Corvallis, OR: AUAI Press.

Lewis, D. 1973. Counterfactuals. Cambridge, MA: Harvard University Press.

Neyman, J. 1923. Sur les applications de la thar des probabilities aux experiences Agaricales: Essay des principle.
Pearl, J., and Mackenzie, D. 2018. The Book of Why. New York: Basic Books.

Pearl, J., and Robins, J. M. 1995. Probabilistic evaluation of sequential plans from causal models with hidden variables. In Besnard, P., and Hanks, S., eds., Uncertainty in Artificial Intelligence 11. San Francisco: Morgan Kaufmann. 444-453.

Pearl, J. 1993a. Aspects of graphical models connected with causality. Proceedings of the 49th Session of the International Statistical Institute 1(August):399-401.

Pearl, J. 1993b. Graphical Models, Causality, and Intervention. Statistical Science 8(3):266-273.

Pearl, J. 1994. A probabilistic calculus of actions. In Uncertainty in Artificial Intelligence 10. San Mateo, CA: Morgan Kaufmann. 454-462.

Pearl, J. 1995. Causal diagrams for empirical research. Biometrika 82(4):669-688.

Pearl, J. 2000. Causality: Models, Reasoning, and Inference. New York, NY, USA: Cambridge University Press, 2nd edition.

Pearl, J. 2010. Review of N. Cartwright 'Hunting Causes and Using Them'. Economics and Philosophy 26:69-77.

Rubin, D. B. 1974. Estimating causal effects of treatments in randomized and nonrandomized studies. Journal of Educational Psychology 66(5):688-701.

Shpitser, I., and Pearl, J. 2006a. Identification of Conditional Interventional Distributions. In Proceedings of the Twenty-Second Conference on Uncertainty in Artificial Intelligence, 437-444. Corvallis, OR: AUAI Press.

Shpitser, I., and Pearl, J. 2006b. Identification of Joint Interventional Distributions in Recursive semi-Markovian Causal Models. In Proceedings of the Twenty-First AAAI Conference on Artificial Intelligence, volume 2, 1219-1226.

Shpitser, I., and Sherman, E. 2018. Identification of Personalized Effects Associated With Causal Pathways. In Proceedings of the 34th Conference on Uncertainty in Artificial Intelligence, 530-539.

Spirtes, P.; Glymour, C. N.; and Scheines, R. 1993. Causation, Prediction, and Search. New York: Springer-Verlag.

Spirtes, P.; Glymour, C. N.; and Scheines, R. 2001. Causation, Prediction, and Search. MIT Press, 2nd edition.

Strotz, R. H., and Wold, H. O. A. 1960. Recursive versus nonrecursive systems: An attempt at synthesis. Econometrica 28:417-427.

Tian, J., and Pearl, J. 2002a. A General Identification Condition for Causal Effects. In Proceedings of the Eighteenth National Conference on Artificial Intelligence (AAAI 2002), 567-573. AAAI Press/The MIT Press.

Tian, J., and Pearl, J. 2002b. On the Testable Implications of Causal Models with Hidden Variables. Proceedings of the Eighteenth Conference on Uncertainty in Artificial Intelligence (UAI-02) 519-527.

Tian, J. 2004. Identifying Conditional Causal Effects. In Proceedings of the 20th Conference on Uncertainty in Artificial Intelligence, UAI '04, 561-568. Arlington, Virginia, United States: AUAI Press.

Tian, J. 2008. Identifying Dynamic Sequential Plans. In In Proceedings of the Twenty-Fourth Conference Annual Conference on Uncertainty in Artificial Intelligence (UAI-08), 554-561. Corvallis, Oregon: AUAI Press.

Woodward, J. 2003. Making Things Happen. New York, NY: Oxford University Press. 